\title{
Survey on Color Image Enhancement Techniques using Spatial Filtering
}

\author{
S. Abdul Saleem \\ Research Scholar \\ Bharathidasan University, Trichirappalli \\ Tamil Nadu, India
}

\author{
T. Abdul Razak, Ph.D \\ Associate Professor in Computer Science \\ Jamal Mohamed College, Trichirappalli \\ Tamil Nadu, India
}

\begin{abstract}
Image Enhancement is one of the major research areas in digital image processing. The main intention of image enhancement is to process the image so that the result is more compatible than the original image for a specific application. Many images like satellite images, medical images, aerial images and even our photographs suffer from poor contrast and noises due to various reasons such as lighting, weather or equipment that has been used to capture the image. It is necessary to enhance the contrast and remove the noises to increase the image quality by using image parameters. Image enhancement techniques differ from one field to another according to its objective. The noises such as Gaussian noise, Salt and Pepper noise and Speckle noise affect most of the images. This paper discusses the advantages and disadvantages of various image enhancement techniques and the metrics which have been used for quantitative measures. Finally it decides which techniques are most appropriate for the real-time image enhancement.
\end{abstract}

\section{General Terms}

Image enhancement, frequency domain, spatial domain, histogram processing, contrast enhancement.

\section{Keywords}

Gaussian noise, salt \& pepper noise, speckle noise, image parameters, multispectral images, fuzzy logic.

\section{INTRODUCTION}

Color images provide more information for visual perception than that of gray scale images. The color image enhancement plays an important role in digital image processing. It is wellknown that image enhancement as an active topic in the field of medical imaging and remote sense imaging. Our principle aim is to improve the visual appearance of the image or to provide better transform for future automated image processing such as analysis, detection, segmentation and recognition[1]. This paper involves the comparative analysis of color image enhancement techniques. The survey of available image enhancement techniques are classified into two broad categories: Frequency domain and spatial domain. The frequency domain methods of image enhancement use the Discrete Fourier Transform(DFT), Discrete Wavelet Transform (DWT) and Discrete Cosine Transform (DCT) of the image and modify its transform coefficients such as brightness, contrast or the distribution of color levels for the image enhancement[3]. After that by using inverse Fourier transform the resultant image is obtained. The frequency domain methods can be classified into three categories namely image smoothing, image sharpening and periodic noise reduction by frequency domain filtering. The main limitation of frequency domain methods is that it cannot simultaneously enhance all parts of image very well and it is also difficult to automate the image enhancement procedure. In the case of spatial domain methods, we can directly deal with image pixels. The main advantages of spatial domain methods are that they conceptually simple to understand and the complexity of these techniques are low which favor for real time implementations. But these techniques generally lacks in providing adequate robustness and imperceptibility requirements. The spatial domain methods can be further classified into two categories namely intensity transformation and spatial filter operations. The spatial filter operations may be linear or non-linear. Mean filters are the examples of linear filters[2]. Normally they have the following problems[3]:

1. A single pixel with a very unrepresentative value can extensively affect the mean value of all the pixels in its neighborhood

2. When the filter neighborhood overlaps an edge, the filter will interpolate new values for pixels on the edge and so will blur that edge. This may be a problem if sharp edges are required in the output.

Both of these problems are tackled by the median (non-linear) filters. Particularly median filters preserve the image details significantly. The advantages of median filters over linear filters are[3]:

1. The median filter is a stronger than linear filter and so a single very unrepresentative pixel in a neighborhood will not affect the median value extensively.

2. Since the median value must actually be the value of one of the pixels in the neighborhood, the median filters do not create new idealistic pixel values when the filter overlaps an edge. For this reason the median filters are much better at preserving sharp edges than the mean filters.

Nowadays median filters play the vital role in many image enhancement techniques. In this paper we compare the nonlinear spatial domain based Image Enhancement techniques. This paper is organized as follows:

The section 2 summarizes images and noises, section 3 discusses the frequency domain methods, section 4 discusses spatial domain methods, section 5 gives the comparison of some spatial filters for image enhancement and section 6 gives the conclusion of our work. 


\section{IMAGES AND NOISES}

An image may be defined as a two-dimensional light intensity function, $f(x, y)$, where $x$ and $y$ are spatial coordinates, and amplitude of $\mathrm{f}$ at any pair of coordinates $(\mathrm{x}, \mathrm{y})$ is called the intensity or gray level of the image at that point. When $\mathrm{x}, \mathrm{y}$ and intensity values of $f$ are all finite, discrete quantities, we call the image a digital image[1].

\subsection{Types of Digital Images}

Digital images may be of four basic types : Binary images, Indexed or Pseudo color images, Grayscale images and True color images.

\subsubsection{Binary Images}

Pixels in Binary images assume one of only two discrete values: 0 or 1 . Figure 1 . shows a binary image with values in specific area[2].

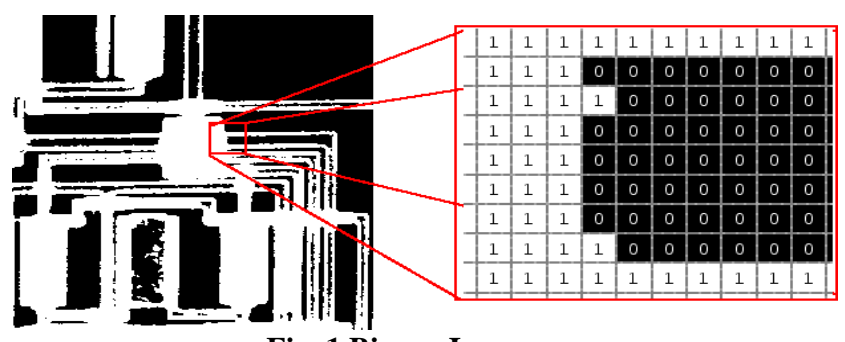

Fig. 1 Binary Image

\subsubsection{Indexed of Pseudo color Images}

Pixels of Indexed Images are direct indices in to a color map which is an $3 \times 3$ matrix containing floating point values in the range $[0,1]$. Figure 2 shows an example of Indexed Pseudo color image[2].

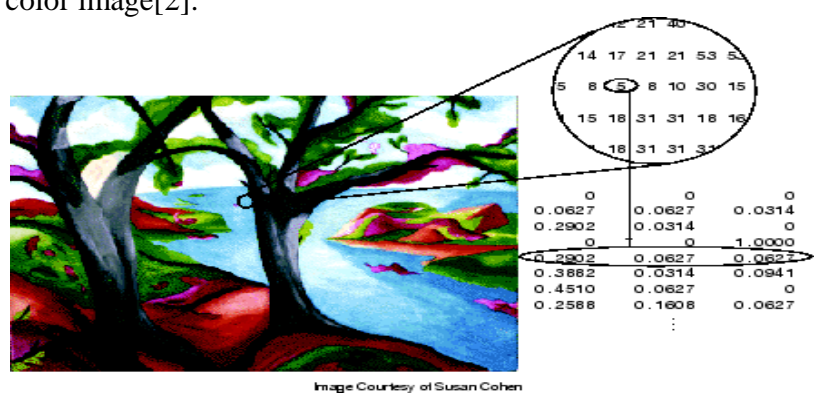

Fig. 2 Indexed Pseudo color image

\subsubsection{Gray-scale Images}

These images are data matrices, whose values represent varying intensities of black and white colors. Figure 3 shows an example of Gray-scale image[3].

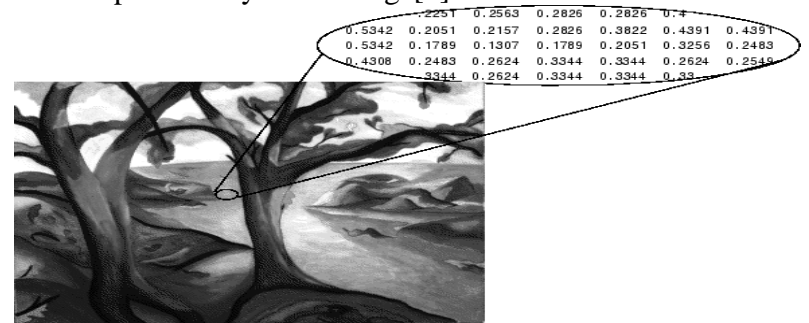

Fig. 3 Gray-scale image
Each pixel in a true color images are specified by 3 valuesone each for the red, green and blue components of the pixel's color. Figure 4 shows a true color image[3].

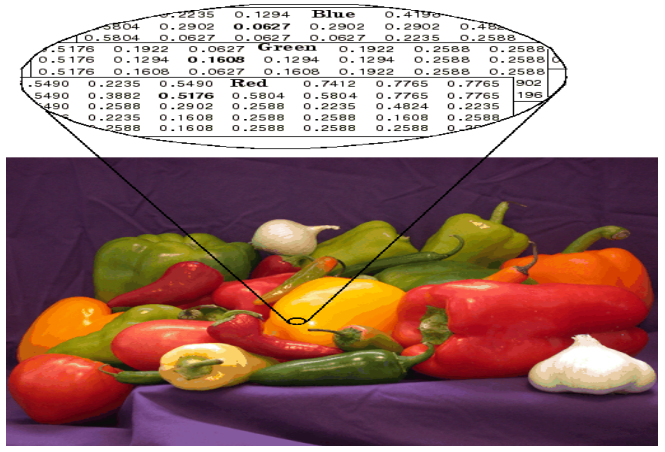

Fig. 3 True Color Image

\subsubsection{Multispectral images}

Multispectral images are the most important type of images acquired by remote sensing sensors. Usually, satellites have three or more image sensors. Each one acquires one digital image in a small band of visible spectra, ranging from $0.7 \mu \mathrm{m}$ to $0.4 \mu \mathrm{m}$, called red-green-blue(RGB) region, and going to infrared wavelength of $0.7 \mu \mathrm{m}$ to $10 \mu \mathrm{m}$ or more classified as near infrared(NIR), middle Infrared (MIR) and far infrared (FIR). In the Landsat case, the seven scenes comprise a sevenband multispectral image. Figure 5 shows an example of Multispectral image[3]. Spectral images with more band, finer spectral resolution or wider spectral coverage may be called hyper spectral.

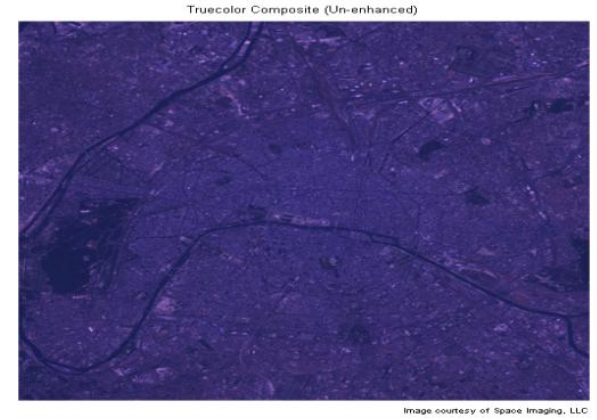

Fig. 5 Multi-spectral image

\subsection{Noises}

Noise is the random variation of brightness or color information in images that contaminates image details. In the image acquisition process, the optical image is converted into continuous electrical signal by the sensor and circuitry of a scanner or digital camera, then it is sampled. During this primary process noises appear in digital image[4]. Image noises are commonly classified into three major types: Random variation impulse noise or Gaussian noise, salt and pepper noise and speckle noises.

\subsubsection{Random Variation Impulse Noise(RVIN)}

RVIN or Gaussian noise occurs randomly as white intensity values. The probability density function of RVIN is,

$$
\mathrm{P}(\mathrm{x})=\frac{1}{(\sigma \sqrt{2 \pi})} * \mathrm{e}^{-\frac{(\mathrm{x}-\mu)^{2}}{2 \sigma^{2}}}
$$

Where, $\mu$ and $\sigma$ are mean and standard deviation of neighborhood pixels values of an image.

\subsubsection{True Color Images}




\subsubsection{Salt and Pepper Noise (SPN)}

It is the random occurrence of both black and white intensity values on the image. It is often caused by threshold of noise image. The probability density function of SPN is

$$
\operatorname{Psp}(X)=\left\{\begin{array}{cc}
P a & \text { for } x=a(" p e p p e r ") \\
P b & \text { for } x=b(\text { "salt") } \\
0 & \text { otherwise }
\end{array}\right\}
$$

Where $P_{\mathrm{a}}$ and $P_{\mathrm{b}}$ are probability density functions; a and b are minimum and maximum intensity values in a kernel or window.

\subsubsection{Speckle Noise (SPN)}

Speckle noises are multiplicative. They occur normally on remote sensing images. The Gaussian noise increases or decreases the brightness of image, where as the Salt and Pepper noise produces black and white dots on the images. These noises are uniform in nature, but the Speckle noise is non uniform and it produces big patches on images.

\section{FREQUENCY DOMAIN METHODS}

Frequency domain methods are based on the manipulation of the orthogonal transform of the image rather than the image itself. These methods are suited for processing the image according to the frequency content. The principle behind the frequency domain method of image enhancement consists of computing the two dimensional discrete unitary transform of the image. For instance two dimensional DFT, manipulating the transform coefficients by an operator $\mathrm{T}$, and then performing the inverse transform. The orthogonal transform of the image has two components namely magnitude and phase. The magnitude consists of the frequency content of the image. The phase is used to restore the image back to the spatial domain. The usual orthogonal transforms are DCT, DFT etc. The transform domain enables operation on the frequency content such as edges and other useful information can easily be enhanced[1].

Frequency domain which operate on the Fourier transform of an image.

1. Edges and sharp transition( eg. Noise) in an image contribute significantly to high-frequency content of Fourier transform.

2. Low frequency contents in the Fourier transform are responsible to the general appearance of the image over smooth areas.

The concept of filtering is easier to visualize in the frequency domain. Therefore enhancement of image $f(x, y)$ can be done in the frequency domain based on DFT is

$$
g(x, y)=h(x, y) * f(x, y)
$$

where $g(x, y)$ is enhanced image. Figure 6 . shows the block diagram of frequency domain method of image enhancement.

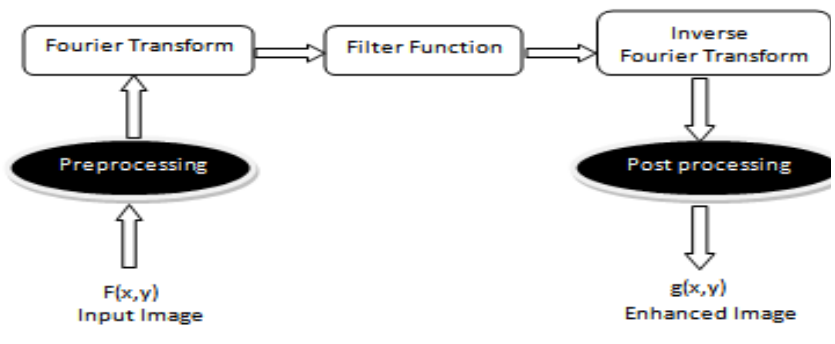

Fig. 6 Image Enhancement in frequency domain

\section{SPATIAL DOMAIN METHODS}

Spatial domain methods directly operate on image pixels. Two principal categories of spatial processing are intensity transformation and spatial filtering. The intensity transformations operate directly on single pixels of an image principally for the purpose of contrast manipulation and image thresholding. Spatial filtering is dealt with performing the operations such as image sharpening and image smoothing.

The Spatial techniques are particularly useful for directly altering the gray level values of the individual pixels and hence the overall contrast of the entire image enhanced. But this uniform enhancement of image pixels can produces undesirable results. It is very tedious to selectively enhance edges and other image details. The spatial domain process can be defined as $\mathrm{g}(x, y)=T[f(x, y)]$, where $\mathrm{f}(\mathrm{x}, \mathrm{y})$ is an input image, $g(x, y)$ in an enhanced image and $\mathrm{T}$ is an operator on $\mathrm{f}$ defined over the neighborhood of point $(x, y)$. The operator can apply to a single image or to a set of images, such as performing the pixel-by-pixel sum of a sequence of images for noise reduction.

\subsection{Intensity Transformation Functions}

The intensity transformation functions include image negative, logarithmic transformation, power law transformation and piecewise linear transformation functions such as contrast stretching, Intensity level slicing and Bitplane slicing are operate single pixels on the image. The histogram processing such as histogram equalization, histogram specification and histogram statistics are also based on the direct manipulation of the pixels in the image for contrast enhancement[1].

The image negative method is suitable for enhancing white or gray details embedded in dark regions of an image. It is given by an expression $\mathrm{s}=\mathrm{L}-1-\mathrm{r}$ where $\mathrm{r}$ and $\mathrm{s}$ are pixel values of before and after processing.

The logarithmic transformation maps a narrow range of low gray levels in to wider range of gray levels. It is given by an expression $\mathrm{s}=\mathrm{c} \log (1+\mathrm{r})$, where $\mathrm{c}$ is a constant and it is assumed that $r>=0$. This transformation helps us to expand the values of dark pixels in an image while compression the higher level values. The opposite is true for the inverse log transformation.

The power law (gamma) transformation have the basic form $\mathrm{s}=\mathrm{c} \mathrm{r}^{\gamma}$ where $\mathrm{c}$ and $\gamma$ are positive constants. If $\gamma<1$, power law transformation maps a narrow range of dark pixel into a wide range and wider ranges of bright pixel values into a narrow range. A variety of devices used for image capture, printing and display respond according to power law[1].

Contrast stretching is a process that expands the range of intensity level in an image so that it spans the dynamic intensity range of the recording medium or display device[5]. The dynamic range is the range between the $I_{\min }$ value and $I_{\max }$ value of an image.

$$
I^{\prime}(x, y)=\frac{d}{\left(I_{\max }-I_{\min }\right)\left(I(x, y)-I_{\min }\right)+I_{0}}
$$

Intensity-level slicing is the process of highlight a specific range of intensities and reduces or preserves other intensities in an image. 
Instead of highlighting intensity-level ranges, we can highlight the contribution made to total image appearance by specific bits. This is known as Bit-plane slicing. This technique determines the capability of number of bits used to quantize each pixel in image compression.

\subsection{Histogram Processing}

Histograms are the basis for most of the spatial domain processing techniques. These are popular technique for realtime image processing. The histogram of an image with intensity level in the range $[0, \mathrm{~L}-1]$ is a discrete function

$$
h\left(r_{k}\right)=n_{k} \text { or } p\left(r_{k}\right)=n_{k} / M N, k=0,1, \ldots, L-1
$$

Histogram Equalization(HE) is the one of the most commonly used methods for image enhancement. This method alters the spatial histogram of an image to closely match a uniform distribution. In general, HE can be divided into three types, Global Histogram Equalization(GHE), Adaptive Histogram Equalization(AHE) and Block-based Histogram Equalization(BHE). In GHE, each pixel is assigned a new intensity value based on previous cumulative distribution function[5].

$$
I^{\prime}(x)=\frac{d}{\operatorname{Cmax}-\mathrm{Cmin}} x\left(C(x)-I_{\min }\right)+I_{0}
$$

Where, I' $(x)$ is the new intensity level, $d$ is the new dynamic range value, $I_{0}$ is the offset point of new dynamic range for $I^{\prime}(x), C(x)$ is the normalized cumulative value and $C_{\max } \&$ $\mathrm{C}_{\min }$ are maximum and minimum values in normalized cumulative value respectively.

The advantages of HE include uniform distribution of intensity values, optimal contrast enhancement and fast processing. The disadvantage is that it is not suitable property in some applications such as consumer electronic products, where brightness preservation is necessary to avoid annoying artifacts. It results in undesired loss of visual data of quality and of intensity scale.

Histogram Specification is another method for contrast enhancement. In this method the shape of the histogram is specified manually and then a transformation function is constructed based in this histogram input image at gray level.

Histogram Statistics obtained directly from an image histogram can be used for image enhancement. The global mean and variance are two components computed over an entire image and are useful for gross adjustments in overall intensity and contrast.

\subsection{Spatial Filtering}

Filtering is a mathematical operation in which intensity of one pixel is combined with intensity of neighboring pixels. This neighborhood is defined by a box that has at least three pixels on a side.

The spatial filters may be linear or non-linear. Mean filters are linear filters which are reducing the amount of intensity variations between one pixel and the next including itself. Median filters are non-linear filters, they simply replacing the pixel value with the median of neighboring pixel values. Median filters are known for their capability to remove impulse noise as well as preserve the edges of images[1].

\subsubsection{Mean Filters}

The simplest form of mean filter sums the intensity of pixels in the kernel and determines the mean. The averaging of neighboring pixels will result the blurred image due to the random nature of pixels at the neighboring positions.

\subsubsection{Adaptive Weighted Mean Filter(AWMF)}

To avoid the blurring on images in linear filter, a new AWMF was introduced. This filter introduces the adaptive combination of pixel values surrounding the pixel $I(i, j)$. Figure 7 shows a kernel or window for an adaptive weighted median filter. The given image is passed through an edge detector and converted into a binary image. If the pixel of interest $I(i, j)$ is an edge, the particular pixel is left unchanged. If the pixel of interest $I(i, j)$ is not an edge, adaptive weighted mean filter omitting the edge pixel is applied[3].

\begin{tabular}{|l|l|l|}
\hline $\mathbf{1}$ & $\mathbf{2}$ & $\mathbf{1}$ \\
\hline $\mathbf{2}$ & $\mathbf{4}$ & $\mathbf{2}$ \\
\hline $\mathbf{1}$ & $\mathbf{2}$ & $\mathbf{1}$ \\
\hline
\end{tabular}

Fig. 7 Kernel or window for adaptive weighted mean filter

\subsubsection{Edge Adaptive Sigma Mean Filter}

The sigma filter averages only those pixels with in a box of predetermined size that do not deviate too much from the pixel that the box is centered on. To set the threshold value for a sigma filter, one must know the type of noise affecting the image and its standard deviation. To avoid this problem edge adaptive sigma filter introduced for contrast enhancement. In this technique, the given image is passed into edge detector and converted in to a binary image. If the pixel of interest $\mathrm{I}(\mathrm{x}, \mathrm{y})$ is an edge, the high pass filter mask is applied to sharpen the edge. But the pixel of interest $I(x, y)$ is not an edge, the low pass mean filter omitting the edge is applied[3].

\subsubsection{Edge Adaptive Hybrid Filter (EAHF)}

To improve the edges and fine details present in the given image, EAHF combines the low pass and high pass filters. This algorithm is also an edge detector, is applied to a given image and converted in to binary image. If the pixel of interest $\mathrm{I}(\mathrm{i}, \mathrm{j})$ is an edge. A high pass filter mask is applied to sharpen the edge. If the pixel of interest $I(i, j)$ is not an edge, a low pass mean filter is applied to improve the fine details[3].

\subsubsection{Median Filters}

Median filters are also called order statistic filters. They remove the isolated pixels in an image effectively, whether they are bright or dark. The popular median filters come to existence are given below.

The Standard Median Filter(SMF) is a non linear, low-pass filtering method which can be used to remove speckle noise from an image.

$$
f(i, j)=\operatorname{median}_{(k, l)} \in W_{h, w}\{D(i+k, j+l)\}
$$

where, $\mathrm{W}_{\mathrm{h}, \mathrm{w}}$ is a sliding window of size $\mathrm{h} \mathrm{x}$ w pixels centered at coordinate $(\mathrm{i}, \mathrm{j})$. This filter doesn't differentiate between uncorrupted and corrupted pixels. Furthermore SMF requires large kernel size if the noise density in high. But the large kernel size will introduce a significant distortion in to the image[1].

The Weighted Median Filter(WMF) was first introduced by Justusson in 1981 and further extended by Brownrigg. It is similar to SMF, except that WMF has weight associated with each of its filter element. These weights are corresponds to the 
number of sample duplications for the calculation of median value[6]. The WMF is given by the expression

$$
f(i, j)=\operatorname{median}_{(k, l) \in W_{h, w}}\left\{W_{h, k}(k, l) \Theta D(i+k, j+l)\right\}
$$

Where, $\Theta$ is repetition operator. This filter preserves image details which is highly dependent[6].

The Decision Based Algorithm (DBA) processes the degraded image by first detecting the impulse noise. The noise detection is decided by checking whether the value of a processed pixel element lies between the maximum and minimum values that occur inside the selected window. If the processed pixel value is within the range, then it is an uncorrupted pixel and left unchanged. If the value doesn't lie within this range, then it is noisy pixel and is replaced by the median value of the window or by its neighborhood values. At higher noise densities, the median value may also be a noisy pixel in which case neighborhood pixels are used for replacement. Repeated occurrence of this type of replacement is lead to streaking effect. Since DBA uses simple fixed length window of size $3 \times 3$, it requires significantly lower processing time than other algorithms[13].

The Adaptive median filter (AMF) is a decision based or switching filter that first identifies possible noisy pixels and then replaces them using the median value or its variants and this method leaves all other pixels unchanged. This method is good at detecting noise at high noise densities provided that the window size is large enough[15].

The Modified Trimmed Median Filter(MTMF) is introduced to overcome the drawbacks in SMF,WMF and AMF, which are not suitable for images corrupted with noise level is over $50 \%$ and furthermore they do not preserve the edges. This algorithm uses $3 \times 3$ window for observation[9].

The High Performance Decision based Median Filter(EDBMF) is an enhanced adaptive switching Median filter, which initially detects noise pixels iteratively through several phases and replaces noisy pixels with median value. This method calculates median value without considering noisy pixels to improve the performance of median filter for

\section{COMPARISON OF IMAGE ENHANCEMENT TECHNIQUES}

Table-1 shows the comparison of Histogram techniques and eight different spatial domain median filters for image enhancement. high density noise. This method expands the sliding window size up to $7 \times 7$. Suppose the processing pixel is replaced by last processed pixel and it also noisy, the size of the window is doubled(15x15) and calculate the number of $0 \mathrm{~s}$ and $255 \mathrm{~s}$ in the processing window then replace the processing pixel with 0 or 255 which is more in the selected window. This method gives better performance in comparison with other listed filters by using quantitative metrics such as Peak-SignalNoise-Ratio(PSNR), Mean Square Error(MSE), Image Enhancement Filter(IEF) and Mean Structural Similarity Index Metric (MSSIM)[10].

$$
P S N R=\frac{10 \log 10(M A X) 2}{\left(M S E_{r}+M S E_{g}+M S E_{b}\right) / 3}
$$

$$
M S E=\frac{1}{M N} \sum_{i=0}^{M-1} \sum_{j=0}^{N-1}\left(\left(R(i, j)-R^{\prime}(i, j)\right)^{2}+\left(G(i, j)-G^{\prime}(i, j)\right)^{2}+\left(B(i, j) B^{\prime}(i, j)\right)^{2}\right)
$$

$$
I E F=\frac{\sum_{i=0}^{M} \sum_{j=0}^{N}\left(n_{i, j}-r_{i, j}\right)^{2}}{\sum_{i=0}^{M} \sum_{j=0}^{N}\left(x_{i, j}-r_{i, j}\right)^{2}}
$$

$$
\operatorname{SSIM}=\frac{\left(2 \mu_{\mathrm{x}} \mu_{\mathrm{y}}+\mathrm{C}_{1}\right)\left(2 \sigma_{\mathrm{xy}}+\mathrm{C}_{2}\right)}{\left(\mu_{\mathrm{x}}^{2}+\mu_{\mathrm{y}}{ }^{2}+\mathrm{C}_{1}\right)\left(\sigma_{\mathrm{x}}{ }^{2}+\sigma_{\mathrm{y}}{ }^{2}+\mathrm{C}_{2}\right)}
$$

Where, $\mu_{\mathrm{x}}$ and $\mu_{\mathrm{y}}$ are the averages of $\mathrm{x}$ and $\mathrm{y}$ $\sigma_{\mathrm{x}}{ }^{2}$ and $\sigma_{\mathrm{y}}{ }^{2}$ are variances of $\mathrm{x}$ and $\mathrm{y}$

$\sigma_{x y}$ is the correlation coefficient between $\mathrm{x}$ and $\mathrm{y}$

$\mathrm{C}_{1}$ and $\mathrm{C}_{2}$ are small constants for stabilize the computation.

The Decision Based un-symmetric Trimmed Median Filter(DBUTMF) overcomes the problem of streaking effect. In this method the left and right extreme values of the sorted array obtained from $3 \times 3$ window are impulse values and are trimmed. The corrupted pixel is replaced by the median of the resultant array. The quantitative performance in

\begin{tabular}{|c|c|c|c|c|c|}
\hline S. No & $\begin{array}{l}\text { Author \& } \\
\text { Year }\end{array}$ & $\begin{array}{l}\text { Enhancement } \\
\text { Technique }\end{array}$ & $\begin{array}{l}\text { Quantitative } \\
\text { Metrics }\end{array}$ & Advantages & Disadvantages \\
\hline 1 & $\begin{array}{l}\text { Gabriel } \\
\text { Thomas et.al, } \\
2011\end{array}$ & $\begin{array}{c}\text { Histogram } \\
\text { Specification[7] }\end{array}$ & $\begin{array}{l}\text { RMSE and } \\
\text { PSNR }\end{array}$ & $\begin{array}{l}\text { Image has uniform histogram, } \\
\text { produce optimal contrast, Fast }\end{array}$ & $\begin{array}{l}\text { Cannot adopt the local } \\
\text { information of the image and } \\
\text { preserves the brightness of } \\
\text { the original image }\end{array}$ \\
\hline 2 & $\begin{array}{l}\text { Abdullah-Al- } \\
\text { Wadud .M } \\
\text { et.al., } 2007\end{array}$ & $\begin{array}{c}\text { Dynamic Histogram } \\
\text { Equalization[8] }\end{array}$ & $\begin{array}{l}\text { PSNR, } \\
\text { AMBE }\end{array}$ & $\begin{array}{l}\text { Preserves the image details, } \\
\text { Ensures a moderate contrast } \\
\text { enhancement of each portion of } \\
\text { a whole image. }\end{array}$ & Brightness is not preserved. \\
\hline
\end{tabular}
terms of PSNR, MSE and IEF for all the algorithms along with DBUTMF shows that, it provides good result for high level noise densities.

Table 1. Comparison of image enhancement techniques using spatial filtering 


\begin{tabular}{|c|c|c|c|c|c|}
\hline 3 & $\begin{array}{l}\text { Ms. Seema } \\
\text { Rajput et. al., } \\
2013\end{array}$ & $\begin{array}{l}\text { Edge Adaptive } \\
\text { Hybrid Filters[9] }\end{array}$ & $\begin{array}{l}\text { PSNR,MSE, } \\
\text { CC,SSIM }\end{array}$ & $\begin{array}{l}\text { Preserves sharp edges and } \\
\text { improve the fine details in the } \\
\text { finger-print images }\end{array}$ & $\begin{array}{l}\text { Suitable for low density } \\
\text { images and requires high } \\
\text { computation time }\end{array}$ \\
\hline 4 & $\begin{array}{c}\text { Krishan Kant } \\
\text { Lavania } \\
\text { et.al.,2012 } \\
\end{array}$ & $\begin{array}{l}\text { Center-to-Boundary } \\
\text { and Boundary -to- } \\
\text { Boundary Filter[11] }\end{array}$ & $\begin{array}{l}\text { EME,MSE,R } \\
\text { MSE, PSNR }\end{array}$ & $\begin{array}{l}\text { Better than average filters, } \\
\text { Enhance the poor quality } \\
\text { images very effectively }\end{array}$ & $\begin{array}{l}\text { Decreases the quality of } \\
\text { image when the filter } \\
\text { dimension is increased }\end{array}$ \\
\hline 5 & $\begin{array}{l}\text { Browning D. } \\
\text { R. K., } 1984\end{array}$ & $\begin{array}{l}\text { Weighted Median } \\
\text { Filter[16] }\end{array}$ & $\begin{array}{l}\text { PSNR,MSE, } \\
\text { MAE }\end{array}$ & $\begin{array}{l}\text { Preserves image details is } \\
\text { highly dependent on the } \\
\text { weighting coefficient. }\end{array}$ & $\begin{array}{l}\text { Finding suitable weighing } \\
\text { coefficient is very difficult In } \\
\text { practical and it takes High } \\
\text { computation time }\end{array}$ \\
\hline 6 & $\begin{array}{l}\text { Nair Madhu } \\
\text { S. et.al., } 2008\end{array}$ & $\begin{array}{c}\text { Decision Based } \\
\text { Algorithm[13] }\end{array}$ & $\begin{array}{l}\text { PSNR, MSE, } \\
\text { SSIM,IEF }\end{array}$ & $\begin{array}{l}\text { Better than SMF,AMF and } \\
\text { other noise removal algorithms. } \\
\text { Suitable for high density noisy } \\
\text { images }\end{array}$ & $\begin{array}{l}\text { Computation Complexity, } \\
\text { Streaking Effect and requires } \\
\text { more computation Time }\end{array}$ \\
\hline 7 & $\begin{array}{l}\text { Hwang H } \\
\text { et.al., } 1995\end{array}$ & $\begin{array}{l}\text { Adaptive Median } \\
\text { Filter[15] }\end{array}$ & PSNR, MSE & $\begin{array}{l}\text { It performs well at low noise } \\
\text { density } \\
\text { Easy to implement }\end{array}$ & $\begin{array}{l}\text { At high noise level it } \\
\text { removes image features }\end{array}$ \\
\hline 8 & $\begin{array}{l}\text { Kwame Oaei } \\
\text { Boating } \\
\text { et.al.,2012 }\end{array}$ & $\begin{array}{l}\text { Effective Median } \\
\quad \text { Filter[17] }\end{array}$ & $\begin{array}{l}\text { PSNR and } \\
\text { MSE }\end{array}$ & $\begin{array}{l}\text { Better than Std. and Adaptive } \\
\text { Median Filters when the noise } \\
\text { density is up to } 50 \%\end{array}$ & $\begin{array}{l}\text { Not suitable for high density } \\
\text { noises }\end{array}$ \\
\hline 9 & $\begin{array}{c}\text { Saravana } \\
\text { Lakshmi C.H. } \\
\text { et. al, } 2012 \\
\end{array}$ & $\begin{array}{l}\text { Modified Trimmed } \\
\text { Median Filter[14] }\end{array}$ & PSNR,IEF & $\begin{array}{l}\text { Better than SMF, AMF, DBA } \\
\text { and MDBA } \\
\text { Good for high noise density }\end{array}$ & $\begin{array}{l}\text { Doesn't preserves edges and } \\
\text { requires more computation } \\
\text { time }\end{array}$ \\
\hline 10 & $\begin{array}{c}\text { Golam } \\
\text { Moktader } \\
\text { Daiyan et. al., } \\
2012\end{array}$ & $\begin{array}{l}\text { High Performance } \\
\text { Decision Based } \\
\text { Median Filter[18] }\end{array}$ & $\begin{array}{l}\text { PSNR, } \\
\text { MSE,IEF and } \\
\text { MSSIM }\end{array}$ & $\begin{array}{l}\text { Expandable filter window size } \\
\text { Less computation time }\end{array}$ & $\begin{array}{l}\text { Loses image features at noise } \\
\text { density more than } 50 \%\end{array}$ \\
\hline 11 & $\begin{array}{l}\text { Aiswarya K. } \\
\text { et.al., } 2010\end{array}$ & $\begin{array}{l}\text { Decision Based Un } \\
\text { Symmetric } \\
\text { Trimmed Median } \\
\text { Filter[19] }\end{array}$ & $\begin{array}{l}\text { PSNR, MSE, } \\
\text { and IEF }\end{array}$ & $\begin{array}{l}\text { Better than Std. and DBAs } \\
\text { when the noise density ranging } \\
\text { from } 10 \text { to } 90 \text {. The MS Sorting } \\
\text { algorithm reduces the } \\
\text { computation time and increases } \\
\text { the efficiency of the system. } \\
\text { Preserves the edges and fine } \\
\text { details. }\end{array}$ & Computation complexity \\
\hline 12 & $\begin{array}{l}\text { Soruba } \\
\text { Marcel J. } \\
\text { et.al.,2011. }\end{array}$ & $\begin{array}{l}\text { Adaptive Fuzzy } \\
\text { Switching Weighted } \\
\text { Median Filter[20] }\end{array}$ & $\begin{array}{l}\text { PSNR, MAE } \\
\text { and } \\
\text { Processing } \\
\text { Time } \\
\end{array}$ & $\begin{array}{l}\text { Fuzzy Logic } \\
\text { initialization of } \text { Based } \\
\text { window size and precision of } \\
\text { median value }\end{array}$ & $\begin{array}{l}\text { Take more computation time } \\
\text { even for low noise densities. }\end{array}$ \\
\hline
\end{tabular}

\section{CONCLUSION}

In this paper, the histogram techniques and eight common median filtering techniques have been presented. Each technique has its own advantages and disadvantages. Some techniques combine the existing two techniques and enhance the existing methods for multiplicative noise removal and edge preservation. However identifying the noisy pixel and efficient replacement of noisy pixel by median value is insufficient for real-time processing. Our aim of image enhancement is to modify the attributes of an image to make it more suitable for a given task and a specific observer. Fuzzy Logic provides a simple and easy way to draw a definite conclusion from ambiguous, imprecise or vague information. It is more similar with the way of human thinking which generate solution from the expertise experiences (represent in rules), knowledge and even skill. The fuzzy based modification of image attributes and enhancement of color images is an application of fuzzy in the area of color feature extraction. Since the enhancing images and preserving image details are the important task of real-time image processing, the adaptive fuzzy logic based noise detection and correction techniques are suitable for real-time color image enhancement.

\section{REFERENCES}

[1] Rafael C. Gonzalez and Richard E. Wood, 2009. "Digital Image Processing”, $3^{\text {rd }}$ Edition, Prentice-Hall.

[2] The Mathwork website: Image processing tool box. http://www.mathwork.com/access/helpdesk/hel/toolbox/ images, viewed on November 2013.

[3] Fishers, R. Perkins, Walker, S. and Wolfart, E, 2003. Web document available: http://homepages. inf.ed.ac.uk/ rbf/ HIPR2/ mean.htm.

[4] Salem Saleh Al-amri and Dr Khamitkar, S.D. 2010. "A Comparitive Study of Removal Noise from Remote Sensing Image", International Journal of Computer Science (IJCSI), ISSN: 1694-0814,vol. 7, no. 1.

[5] Anish Kumar Vishwakarma and Agya Mishra, 2012. "Color Image Enhancement Techniques: A Critical Review”,ISSN:0976-5166,vol. 3, no.1,pp.39-45.

[6] Sin Hoong Teoh and Haidi Ibrahim, 2012. "Median Filtering Frameworks for Reducing Impulse Noise from Gray Scale Digital Images: A Literature Survey", International Journal of Future Computer and Communication, vol. 1, no. 4. 
[7] Gabriel Thomas, Daniel Flores-Tapia and Stephen Pistorius, 2011. "Histogram Specification : A fast and Flexible Method to process Digital Images" IEEE Trans. On Instrumentation and Measurement, vol. 60 , no. 5 , pp. $1565-1578$.

[8] Wadud Kair, A.A. Dewan M.H Dewan and Oksam, M.C. 2007. "A Dynamic Histogram Equalization for Image Contrast Enhancement", vol. 53, no. 2, pp. 593-600, IEEE Trans.

[9] Ms. Seema Rajpu and Suralkar, S.R. 2013. "Comparitive Study of Image Enhancement Techniques", vol. 1, ISSN 2320-088X, pp. 11-21, Intenational Journal of Computer Science and Mobile Computing(IJCSMC).

[10] Huang, T., Yang, G. and Tang, 1979. "A Fast TwoDimensional Median Filtering Algorithm", IEEE Trans., Acoust., Speech, Signal Processing, vol. 27, no. 1,pp. 13-18.

[11] Krishan Kant Lavania, Shivali and Rajiv Kumar, 2012. "Image Enhancement using Filtering Techniques", International Journal of Computer Science and Engineering(IJCSE), vol. 4, no. 1.

[12] Srinivasan, E. and Ebenezer, D., 2008. "New Nonlinear Filtering Strategies for eliminating short and long tailed image preservation properties" International Journal of Information and Communication Engineering, vol. 4, no. 3, pp. 175181.

[13] Nair Madhu, S., Revathy, K. and Tatavarti Rao, 2008. "Removal of Salt and Pepper Noise in Images: A new Decision-Based Algorithm "Proceeding of
International Multi Conference of Engineers and Computer Scientists(IMECS), vol. 1, pp. 19-21.

[14] Saravana Lakshmi, C.H., ,Ambika, V. and Suri Babu, K. 2012. "Impulse Noise Removal using Modified Trimmed Median Filter", International Journal of Engineering Research and Applications,vol. 2, pp. 2163-2166, ISSN 2248-9622.

[15] Hwang, H. and Hadded, R.A., 1995. "Adaptive Median Filter: New algorithms and results", IEEE Trans. Image Processing, vol. 4 no. 4, pp. 499-502.

[16] Browning, D. R. K.,1984. "The Weighted Median Filter" Communication, ACM, vol. 27, no. 8, pp. 807-818.

[17] Kwame Osei Boating, Benjamin Weyori Asubam and David Sanka Laar, 2012. "Improving the Effectiveness of the Median Filter", International Journal of Electronic and Comunication Engineering, ISSN 0974-2166, vol. 5, pp. 8597.

[18] Golam Moktadar Daiyan, M.A. Mottalib and Muhammed Mizanur Rahman, 2012. "High Performance Decision Based Median Filter for Salt and Pepper Noise Removal in Images" 0978-1-4673-4836-2, IEEE.

[19] Aiswarya, K., Jayaraj, V. and Ebenezer, D. 2010. "A New and Efficient Algorithm for the Removal of High density Salt and Pepper Noise in Images and Videos", 978-0-76953941, IEEE.

[20] Soruba Marcell, J., Jayachandran, A. and . Kharmega Sundararaj, G. 2011. "An efficient algorithm for removal of impulse noise using Adaptive Fuzzy Switching Weighted Median Filter" International Journal of Computer Technology and Electronics Engineering(IJCTEE), vol. 2, ISSN 2249-6343. 